\title{
Dean, Chairs, and Faculty: A Team Approach for Enhancing Faculty Diversity
}

\author{
Simon J. Rhodes
}

N. Douglas Lees

Diversifying the faculty at many colleges and universities has become a significant priority that is featured in strategic plans or in freestanding diversity plans. There are many reasons for this focus, the most directly relevant of which is that faculty diversity plays a key role in creating an inclusive and welcoming environment in our classrooms for students from underrepresented groups. With the demographic shift in the United States to significantly higher percentages of high school graduates who are members of underrepresented groups, the need for diverse faculty in our college and university classrooms is compelling. Related to higher education efforts to diversify its faculties in order to provide role models and a positive classroom atmosphere for minority students is the influence that diversity has on our economy. Employers also see the demographic shift in our population and realize that they will be hiring more graduates from underrepresented groups. Further, business and academic research studies suggest that more diverse teams achieve improved outcomes (Freeman and Huang 2015; Hunt, Layton, and Prince 2015), and employers want an educated and diverse workforce in order to be competitive. Thus, their employee pipeline depends on higher education's ability to prepare all graduates for workplaces that will be more diverse.

The objective here is to report on an approach to diversify the faculty of the School of Science at an urban, public, research university. The School of Science at Indiana University-Purdue University Indianapolis (IUPUI) is comprised of seven departments (biology, chemistry and chemical biology, computer and information science, earth sciences, mathematical sciences, physics, and psychology) and two programs (forensic and investigative sciences and neuroscience). All departments are STEM

This is the author's manuscript of the article published in final edited form as:

Rhodes, S. J., \& Lees, N. D. (2017). Dean, Chairs, and Faculty: A Team Approach for Enhancing Faculty Diversity. The Department Chair, 27(3), 11-13. https://doi.org/10.1002/dch.30119 
departments, and they, like most others, collectively struggle with faculty diversity. At the department level, the lack of diversity reveals itself in ethnicity across the board and in gender selectively.

Because faculty are hired via search processes that take place in academic departments, the department chair's role is critical in assuring that diversity is granted the appropriate consideration. At the other end of the spectrum, the institution has diversity goals but how that message effectively penetrates academic departments is highly variable. IUPUI is a large, complex institution that practices responsibility-centered management. This practice creates an increased sense of autonomy for the schools; they decide when to hire faculty and pay the costs themselves. Accountability for diversity efforts and outcomes takes place at meetings between the campus Office of Equal Opportunity and each school's dean. In order to align diversity goals and to create a real connection between those goals and faculty searches, the model here involves collaborative efforts between the dean and the chairs in a faculty-owned process.

With this somewhat nonarticulated process, how does one go about assuring that the message has been clearly made and that all parties are following best practices? As a preliminary step, the school established a diversity council comprised of faculty, staff, and students. The council was instrumental in assuring that diversity was appropriately represented in the strategic planning process that followed.

Diversity is a key component of the school's vision and values statements as well as the leading concept (“Ensure student and faculty recruitment initiatives are designed to increase representation of Underrepresented Minorities and women in specific departments/areas and ranks.") under the "Develop" segment of the school's strategic plan. The plan is closely aligned with that of the campus and resulted from the combined efforts of the school's faculty, staff, students, and stakeholders and thus represents a community mandate that we act on all of its elements.

A second preliminary step taken by the dean was to have a conversation with each department chair. This was done in recognition of the critical roles that chairs play in interpreting and predicting faculty responses to change, creating a welcoming and supportive environment for new faculty, and leading the change process. At this meeting, the overall enthusiasm for increasing faculty diversity was ascertained. 
All chairs supported the initiative, some with great vigor, while a few expressed reservations about how some of their faculty would respond. Each discipline has its own culture shaped by tradition, faculty personal histories, and unit leadership. This came as no surprise to the dean. The meetings ended with the dean suggesting the general makeup of the search committee, his expectations of the short list of candidates, and a request to be invited to the convening meeting of the search committee.

At the search committee's first meeting, the dean assured the committee that it would own the search but that there was an expectation from the entire school community, based on the diversity language in the strategic plan, that searches be conducted in ways that will help place the school in a position to achieve its diversity goals. He then discussed what diversity means (ethnicity? gender? etc.) in that department. Next there was a conversation on why faculty diversity is good for our students and their success and the recent research demonstrating that diverse organizations reach better decisions and are more productive and creative.

Next on the agenda was a discussion on actively creating a large and diverse pool of candidates. Faculty are encouraged to identify diverse faculty talent at conferences they attend. Departments are encouraged to bring in potential candidates as seminar speakers. Both types of individuals may receive solicitations to apply for the faculty opening. It was emphasized that this is a responsibility of the entire faculty and not just those serving on the search committee. At the end of this conversation, the dean concluded that he expected the list of those to be invited for interviews to have clear elements of diversity.

Before leaving the committee to its work, the dean provided a copy of the publication by Eve Fine and Jo Handelsman, Searching for Excellence and Diversity: A Guide for Search Committees (2005). This book describes best practices in diversity searches, including lists that search committee members can readily use as quick guides. Also provided are web sources posting similar publications, including one from the Association of American Medical Colleges that covers the science of unconscious bias as related to the search process. Faculty awareness and understanding of their own perceptions and biases are valuable 
assets in this process. The meeting was followed up immediately with an email thanking the committee for the conversation and reiterating its components and priorities.

The following are some recommendations for conducting successful searches that yield excellent and diverse candidates.

- The dean, in collaboration with the department chair, should be actively involved to give the process cross-department continuity on expectations. All, including those resistant to change, will know that the dean and the chair are united in the effort.

- Be persistent on the importance of diversity in hiring. Use faculty-approved documents to demonstrate community support. Involve the entire faculty in the process.

- Provide additional funding to interview a candidate who is a near miss for the interview list. Under special fiscal circumstances, make two offers when a diversity candidate is ranked excellent but behind another candidate(s). The Office of Equal Opportunity may provide a waiver for two hires, and the campus may assume some of the costs in such scenarios.

- Ensure that the search committee reflects diversity. One member from outside the department can provide academic culture diversity. Gender diversity should be present as well as ethnic diversity. The latter may be problematic because of small numbers and the inherent unfairness of routinely pulling these faculty from their faculty work. At some institutions, committee diversity may be represented by inviting individuals from the Office of Equal Opportunity or the campus diversity office to serve.

- Utilize the extensive print and electronic resources and best practices already available for improving diversity hiring goals.

- Be patient. Avoid mandating outcomes. Change is difficult for most, but those who are reluctant will get into the routine, especially when excellent new faculty who reflect diversity come aboard. 
- Allow the committee to use its best collective judgment in ranking the candidates. Respect their decisions.

It is the chair who often guides the process, has strong influence in the room, monitors the department atmosphere, and executes the hiring negotiation. Each department has its own personality, and the chair is in the best position to respond to issues that arise in the process and to come to solutions that are compatible with diversity goals.

The process in place in the School of Science has evolved to the state described here. Although it is difficult to report substantive outcomes over a short period of time, the school appears to be on a positive track. In the five years (2011-2012 through 2015-2016) that faculty searches have been conducted in the School of Science under the present leadership, forty-eight faculty members (thirty-two on the tenure track) have been hired; about 42 percent (37.5 percent on the tenure track) have brought gender or ethnic diversity to the school. In regard to assessing the success of diversity hires relative to standard performance measures (teaching effectiveness, publications, external funding success, and contributions to local and professional service), they are doing at least as well as the other hires in the five-year cohort. This outcome bodes well for future searches in that it provides evidence that one can achieve both diversity and excellence.

We are grateful for the combined efforts and expertise of many colleagues, and we especially thank Drs. Leslie Ashburn-Nardo and Peggy Stockdale.

\begin{abstract}
About the Author
Simon J. Rhodes is dean of the School of Science and professor of biology and N. Douglas Lees is associate dean for planning and finance at Indiana University-Purdue University Indianapolis. Email: srhodes@iupui.edu, nlees@iupui.edu
\end{abstract}

\title{
References
}


Fine, Eve, and Handelsman Jo. 2005. Searching for Excellence and Diversity: A Guide for Search Committees. Madison, WI: Women in Science and Engineering Leadership Institute.

Freeman, Richard B., and Huang Wei. 2015. “Collaborating with People Like Me: Ethnic Coauthorship within the United States.” Journal of Labor Economics 33 (S1): S283-S318.

Hunt, Vivian, Layton Dennis, and Prince Sara. 2015. "Why Diversity Matters.” Accessed August 18, 2016. www.mckinsey.com/business-functions/organization/our-insights/why-diversity-matters. 